\title{
Isolement, Identification Et Activite Antifongique De Deux Sesquiterpenes D'asteriscus Graveolens Subsp. Odorus (Schousb.) Greuter
}

\begin{abstract}
Hakim Alilou
Bouchaib Bencharki

Laboratoire d’Agroalimentaire et Santé, Faculté des Sciences et Techniques, Université Hassan 1er, BP 577 Route de Casablanca, Settat, Maroc.

\section{Mohammed Akssira}

Laboratorie de Chimie Physique et Chimie Bioorganique, FST, Université Hassan II Mohammadia-Casablanca. BP 146, Mohammadia 20800, Maroc.

\section{Lalla Mina Idrissi Hassani}

Laboratoire de Biotechnologies Végétales, Département de Biologie, Faculté des Sciences Université Ibn Zohr, BP 28/S-Dakhla, Agadir 80000, Maroc.

\section{Noureddine Barka}

Laboratoire des Sciences des Matériaux, des Milieux et de la Modélisation (LS3M), Faculté Polydisciplinaire de Khouribga, BP. 145 Khouribga, Morocco

doi: 10.19044/esj.2016.v12n33p112 URL:http://dx.doi.org/10.19044/esj.2016.v12n33p112

\section{Abstract}

Two sesquiterpenes of Asteriscus graveolens subsp. odorus, have been extracted, separated, purified and identified by NMR and IR: the naupliolide and asteriscunolide 3c.

The valuation of these compounds is part of the search for a preventive approach of integrated control to reduce significantly the use of pesticides and especially the fungicide ones. The results revealed a strong inhibitory effect on naupliolide against Botrytis cinerea, Penicillium digitatum and Penicillium expansum dice concentration of 200 ppm. The asteriscunolide 3c showed a remarkable effect against Botrytis cinerea from the concentration of $150 \mathrm{ppm}$.
\end{abstract}

Keywords: Antifungal activity, Asteriscus graveolens subsp. odorus, pathogens fungi, Sesquiterpenes 


\section{Résumé}

Deux sesquiterpènes d'Asteriscus graveolens subsp. odorus ont fait l'objet d'une extraction, séparation, purification et identification par RMN et IR : le naupliolide et l'asteriscunolide 3c.

La valorisation de ces deux molécules s’inscrit dans le cadre de la recherche d’une démarche préventive de lutte intégrée visant à la réduction significative de l'utilisation des pesticides et en particulier les fongicides. Les résultats obtenus ont révélé un fort effet inhibiteur du naupliolide sur Botrytis cinerea, Penicillium digitatum et Penicillium expansum dès la concentration de $200 \mathrm{ppm}$. L'asteriscunolide 3c a montré un effet remarquable sur Botrytis cinerea à partir de la concentration de 150 ppm.

Mots clés: Activité antifongique, Asteriscus graveolens subsp. odorus, Champignons pathogènes, Sesquiterpènes

\section{Introduction}

De nos jours, les produits naturels sont une source importante pour la recherche de nouveaux composés actifs contre de nombreuses maladies. L'utilisation thérapeutique des plantes est partie intégrante des traditions de toutes les cultures. La valorisation médicinale de ces pratiques passe notamment par l'isolement et l'identification de nouvelles molécules.

Au cours des vingt dernières années, une attention considérable a été donnée au genre Asteriscus et principalement aux sesquiterpènes lactones. Les asteriscunolides (3a, 3b, 3c et 3d) ont été isolés à partir des parties aériennes d'Asteriscus aquaticus (San Feliciano et al. 1982 ; 1984 ; 1985a et b), Asteriscus graveolens (El-Dahmy et al. 1985), Asteriscus sericeus (Jakupovic et al. 1987) et Asteriscus vogelii (Rauter et al. 2001). Les asteriscunolides et leurs hydroxyacides libres ont été aussi obtenus de l'extrait de Nauplius intermedius (Bohlmann \& Pauls, 1992). D’autres lactones sesquiterpènes comme l'asteriscanolide et l'aquatolide ont été obtenus à partir de l'extrait d'Asteriscus aquaticus (San Feliciano et al. 1985 a et b, 1989). Asteriscus graveolens a été reconnu pour son activité antimicrobienne et hypoglycémiante (Ahmed, 1992). Quant aux extraits des feuilles et des fleurs d'Asteriscus graveolens subsp. odorus, ils sont reconnus par leurs activités antifongiques contre Penicillium digitatum et Penicillium expansum (Alilou et al. 2007 ; 2013).

Dans le but de rechercher les composés actifs dotés de propriétés antifongiques, nous avons purifié et identifié les sesquiterpènes majoritaires d'Asteriscus graveolens subsp. odorus, selon le protocole présenté par Akssira et al. (2006). La valorisation de ces molécules afin de mieux cerner leurs effets sur les champignons pathogènes de post récolte semble intéressante. À cet effet, deux molécules ont été testées à savoir le 
naupliolide, qui est une nouvelle molécule identifiée par Akssira et al. (2006) et qui n’a été jamais étudiée de point de vue activité biologique, et l'asteriscunolide 3c. Cette dernière est le précurseur de biosynthèse de naupliolide. L’activité antifongique a concerné les champignons : Penicillium digitatum, Penicillium expansum et Botrytis cinerea.

\section{Matériel et méthodes}

\section{Matériel végétal}

L’appareil végétatif d'Asteriscus graveolens subsp. odorus a été récolté de la région d’Agadir durant le mois d'avril (2015) de manière aléatoire. Cette plante constitue un patrimoine local floristique très important qui n'est en grande partie décrit que d'un point de vue botanique. Un spécimen de cette plante a été déposé à l'herbier de la Faculté des Sciences d’Agadir (Université Ibn Zohr, Agadir, Maroc) après identification par le Professeur B. CHEBLI à l'École nationale des sciences appliquées d'Agadir (Maroc). La plante a été ensuite séchée à l'ombre, à l'abri de l'humidité et broyée au moulin électrique jusqu'à obtention d'une poudre.

\section{Extraction et fractionnement}

Après extraction chloroformique de $1 \mathrm{~kg}$ de matériel végétal par soxhlet, un décirrage a été effectué à une température de $-20^{\circ} \mathrm{C}$ pendant une nuit en utilisant le chloroforme et le méthanol bouillant. Ensuite, une extraction par le dichlorométhane a été faite suivi d'un lavage avec une solution de $\mathrm{Na}_{2} \mathrm{CO}_{3}$ à $10 \%$ qui a donné deux phases : une phase organique et une phase aqueuse. Cette dernière a été acidifiée jusqu’à pH=2 ( $\mathrm{HCl}$ à 50\%) puis extraite par $\mathrm{CH}_{2} \mathrm{Cl}_{2}$ pour avoir une phase organique et une phase aqueuse. La phase organique a été lavée avec $\mathrm{NaCl} / \mathrm{H}_{2} \mathrm{O}$, séchée avec $\mathrm{Na}_{2} \mathrm{SO}_{4}$ et évaporée pour avoir une phase organique acide. La phase organique a été aussi le résultat du lavage par $\mathrm{Na}_{2} \mathrm{CO}_{3}$ à $10 \%$. Cette phase a subi le même protocole de la phase organique acide. La phase organique neutre obtenue de cette dernière extraction à l'aide du dichlorométhane a été pesée avant de passer au fractionnement par chromatographie sur colonne.

L'extrait neutre (37 g) a été fractionné sur une colonne ouverte de gel de silice après élution par un mélange hexane-acétate d'éthyle de polarité croissante. La chromatographie sur colonne a donné quatre fractions importantes dont on a récupéré seulement deux, selon la méthode préconisée par Akssira et al. (2006) pour obtenir :

$>\quad$ La fraction 1 (1,37g) éluée avec $\mathrm{CH}_{2} \mathrm{Cl}_{2} / \mathrm{EtOAc}$ (9:1) donnant le naupliolide (20 mg)

$>\quad$ La fraction 2 (3,5 g) éluée avec l'hexane/Et ${ }_{2} \mathrm{O}$ (1:1) pour donner l'asteriscunolide 3c (345,4 mg). 


\section{Identification spectrale des produits isolés}

Les spectres infrarouges ont été effectués après avoir mélangé les produits purifiés dans des plaques de $\mathrm{NaCl}$, par un spectrophotomètre de type Génisis Series FTIR de Mattson.

L'analyse GC-MS a été faite sur un spectromètre du thermomass (modèle trio 1000) couplé à un chromatographe en phase gazeuse. Le chromatographe est équipé d'une colonne capillaire ( $25 \mathrm{~m}$ long $\times 0,25 \mathrm{~mm}$ ). La colonne est programmée à une température de $60^{\circ} \mathrm{C}$ pendant $6 \mathrm{~min}$, avec $5^{\circ} \mathrm{C}$ d'augmentation par min puis portée à $150{ }^{\circ} \mathrm{C}$; il a été maintenu pour 10 min. Le gaz vecteur est l'hélium pulsé à la vitesse de $2 \mathrm{~mL} / \mathrm{mn}$. Les températures de détecteur et de l'injecteur ont été maintenues respectivement à 250 et $225^{\circ} \mathrm{C}$. Le spectromètre de masse a été réalisé en utilisant la gamme 28-400 amu.s- ${ }^{1}$, avec un voltage ionisant de $70 \mathrm{eV}$ et un courant de l'ionisation de $150 \mu \mathrm{A}$.

Les composés ont été identifiés par MS et leurs identités ont été confirmées en comparant leurs indices de rétention relative à C8-C32 nalkanes selon Kovats (1958) et en comparant leurs spectres de masse et le temps de rétention avec ceux de produits de différences ou avec les données déjà décrites dans la bibliothèque NIST ou la banque de donnée informatisée (Adams, 2001).

Les spectres RMN ont été enregistrés sur Varian Gemini 300 et Varian Inova 400. Les déplacements chimiques sont référencés au signal résiduel $\mathrm{CHCl}_{3}$ à $\delta 7,26 \mathrm{ppm}$. Les spectres $\mathrm{RMN}{ }^{13} \mathrm{C}$ ont été référencés à la crête centrale de $\mathrm{CDCI}_{3}$ à $\delta 77,0$ ppm. Les spectres HMBC et COSY ont été enregistrés avec des séquences Varian standard gradient d'impulsion.

\section{Test antifongique}

Le naupliolide et l'asteriscunolide 3c ont été purifiés, identifiés et testés pour leur activité antifongique sur Penicillium digitatum, Penicillium expansum et Botrytis cinerea. Une solution mère des deux molécules a été préparée pour obtenir les concentrations finales 0, 50, 100, 125, 150, 200, 250, 500, 1000 et 2000 ppm qui ont été ajoutées au milieu Potato-DextroseAgar (PDA) stérile. $20 \mathrm{~mL}$ de la solution a été coulée, sous agitation magnétique, dans les boites de Pétri. Un disque de $6 \mathrm{~mm}$ de diamètre de culture jeune de chaque champignon a été déposé au centre de la boite de pétri contenant le milieu de culture et les différentes concentrations des produits purs. L'incubation a été effectuée à une température de $25^{\circ} \mathrm{C} \pm 1^{\circ} \mathrm{C}$ pendant 7 jours. Le pourcentage d'inhibition (PI) a été calculé en utilisant la formule suivante :

$\mathbf{P I}=[(\mathbf{C}-\mathbf{T}) / \mathbf{C}] \times \mathbf{1 0 0}$

$\mathrm{C}$ : Diamètre moyen de 3 répétitions de la croissance mycélienne estimé du champignon testé ou de colonie des spores sur milieu témoin $(\mathrm{Cm})$ 
T : Diamètre moyen de 3 répétitions de la croissance mycélienne ou de colonie des spores de boite, traitées avec l'une des concentrations des sesquiterpènes $(\mathrm{Cm})$.

\section{Isolement de l'agent pathogène}

L’isolement de l'agent pathogène a été effectué à partir des fruits d'agrumes présentant les pourritures causées par Penicillium digitatum, Penicillium expansum et Botrytis cinerea. Le choix de ces derniers est dû aux dégâts causés dans les stations de conditionnements de la région du sud du Maroc. Ces champignons ont été isolés à partir des fruits infectés de la station Soussia (Tassila, Agadir). Des morceaux d'écorce infectés sont détachés est placé au centre du milieu de culture PDA. Les boites ainsi préparées sont incubées à $25^{\circ} \mathrm{C}$ pendant sept jours. Après purification par des repiquages successifs sur PDA, le champignon est isolé puis conservé à $4^{\circ} \mathrm{C}$ sur le même milieu de culture.

\section{Résultats et discussions}

Identification des deux lactones sesquiterpèniques d'Asteriscus graveolens subsp. odorus

\section{Identification du naupliolide}

L'identification du naupliolide a été basée sur le protocole déjà cité par Akssira et al. (2006). Ainsi, ce composé se présente sous forme de poudre blanche amorphe. Il a un pouvoir rotatoire $[\alpha]_{\mathrm{D}}=+80 \quad(\mathrm{c}=0.11$; $\mathrm{CHCl}_{3}$ ). La formule moléculaire de ce composé a été déterminée comme $\mathrm{C}_{15} \mathrm{H}_{18} \mathrm{O}_{3}$ par HREIMS, m/z 246,13 indiquant 7 degrés d'insaturation. Le spectre infrarouge a montré une bande d'absorption à $1765 \mathrm{~cm}^{-1}$ correspondant à la bande d'absorption du groupement carbonyle $(\mathrm{C}=\mathrm{O}) \mathrm{du}$ squelette $\gamma$-lactone et une deuxième bande d'absorption $1660 \mathrm{~cm}^{-1}$ correspondant au groupe cétone à $\alpha, \beta$ - insaturés.

Le spectre ${ }^{13} \mathrm{C}$ RMN du naupliolide a montré la présence de 15 signaux, dont chacune représente le signal de résonance d'un carbone. La présence d'une double liaison trisubstituée qui peut être établie par ceux à 136,2 ppm (singulet) et 138,7 ppm (doublet). Les signaux restants, présentent une multiplicité correspondante à deux $\mathrm{CH}_{3}$, trois $\mathrm{CH}_{2}$, cinq $\mathrm{CH}$ et un carbone quaternaire. Leur valeur de $\delta$ est caractéristique du carbone $\mathrm{sp}^{3}$ non fonctionnalisé. Ainsi, le naupliolide contient trois fonctions insaturées et possède quatre cycles dans sa structure.

Le spectre ${ }^{1} \mathrm{H}$ RMN présente des signaux : à 6,34 ppm $(1 \mathrm{H}, \mathrm{ddq}, \mathrm{J}=$ 8.9, 7.7, et $1.3 \mathrm{~Hz}$ ) caractéristique d'une double liaison trisubstituée conjuguée par le groupe cétone, à $1,84 \mathrm{ppm}(3 \mathrm{H}, \mathrm{s})$ et $1,34 \mathrm{ppm}(3 \mathrm{H}, \mathrm{s})$ caractéristiques de deux groupements méthyle. Par ailleurs, un signal à 4,66 ppm $(1 \mathrm{H}$. d. $J=4,2 \mathrm{~Hz})$ a été attribué à un proton lié à un carbone oxygéné. 
D’autres signaux à savoir $0,57 \mathrm{ppm}(1 \mathrm{H}$, dd, $\mathrm{J}=6.0$ et $8.0 \mathrm{~Hz}), 0,64 \mathrm{ppm}$ $(1 \mathrm{H}$, dd, $\mathrm{J}=6.0$ et $4.0 \mathrm{~Hz})$, et $1,61 \mathrm{ppm}(1 \mathrm{H}$, ddd, $\mathrm{J}=8.4$ et $4.0 \mathrm{~Hz})$ ont été attribués à un cycle cyclopropane. L’interprétation des signaux restants a été faite à l'aide de 2D-RMN, HSQC et ${ }^{1} \mathrm{H}-{ }^{1} \mathrm{HCOSY}$ permettant d'établir la structure partielle du naupliolide. Tous les protons sont liés avec une corrélation directe à l'exception de groupement méthyle à $1,34 \mathrm{ppm}$ dans ${ }^{1} \mathrm{H}$ RMN.

Les corrélations HMBC permettent de joindre le composé C1 au groupement méthyle et de déterminer les liaisons entre le reste des fragments structuraux de ce composé. Ainsi, la structure carbonatée du naupliolide a été établie. La stéréochimie de la double liaison a été déterminée par la corrélation NOE observée pour $\mathrm{H}_{6}$ et $\mathrm{Me}^{-\mathrm{C}_{7}}$ en spectre 1D-NOESY. La configuration relative de six carbones stériogéniques a également été établie par le spectre $1 \mathrm{D}-\mathrm{NOESY}$ et $\mathrm{J}_{\mathrm{H} 2-\mathrm{H} 9}=9.9 \mathrm{~Hz}$, confirmant qu'il n'y a pas de relation entre ces protons. En outre, les corrélations NOE observées pour $\mathrm{H}_{5} \beta \mathrm{H}_{9}$ et $\mathrm{H}_{4} \beta \mathrm{H}_{9}$ sont en accord avec la conformation bateau pour la raie cyclooctène. Ainsi, la caractérisation structurale du squelette tetracyclique sesquiterpènique trouvé est celle du naupliolide (Figure 1). Cela est en conformité avec les résultats obtenus par Akssira et al. (2006).

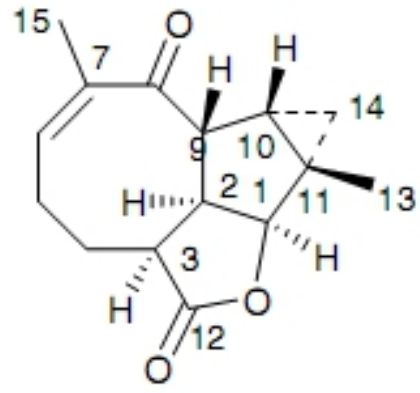

Figure 1 : Structure de naupliolide : 14,15-dimethyl-7,13-dioxotricyclic[6.4.0.0 ${ }^{9,11}$ ]dodeca12,13-olide.

\section{Identification de l'asteriscunolide 3c}

Dans notre étude, nous avons déterminé la structure, la configuration et la conformation du composé $3 c$ par les analyses spectrales ${ }^{1} \mathrm{H}-\mathrm{RMN}$ et ${ }^{13} \mathrm{C}-\mathrm{RMN}$.

Les résultats obtenus ont montré que l'asteriscunolide 3c se présente sous forme de cristal blanc. Au niveau du spectre ${ }^{1} \mathrm{H}-\mathrm{RMN}$, on observe deux doublets à 6,27 et 5,91 ppm, respectivement proposés pour $\alpha$ et $\beta$ des protons $\left(\mathrm{H}_{9}\right.$ et $\left.\mathrm{H}_{10}\right)$. Ces doublets correspondent à des doubles liaisons disubstituées conjuguées à un groupement carbonyle.

Ainsi, les spectres ${ }^{13} \mathrm{C}-\mathrm{RMN},{ }^{1} \mathrm{H}-\mathrm{RMN}$ d'asteriscunolide $3 \mathrm{c}$ sont présentés dans les tableaux 1 et 2 . 
Tableau 1 : Données spectrales ${ }^{1} \mathrm{H}$-RMN d'asteriscunolide 3c

$\begin{array}{lllll}\text { Asteriscunolide 3c } & 5.91(\mathrm{~J}=16.5) & 6.27 \mathrm{~d} & 1.36 & 1.28\end{array}$

Tableau 2 : Données spectrales ${ }^{13} \mathrm{C}$-RMN d'asteriscunolide $3 \mathrm{c}$

\begin{tabular}{ccccccccccccccc}
\hline C1 & C2 & C3 & C4 & C5 & C6 & C7 & C8 & C9 & C10 & C11 & C12 & C13 & C14 & C15 \\
\hline 85,6 & 149,7 & 138,6 & 22,8 & 33,9 & 128,4 & 135,6 & 202,6 & 129,5 & 156,4 & 40,7 & 173,7 & 21,1 & 24,6 & 21,0
\end{tabular}

Ainsi, la caractérisation de la structure d'asteriscunolide 3c (Figure 2) est en accord avec Akssira et al. 2006, San Feliciano et al. (1982 ; 1984 ; 1985a et b).

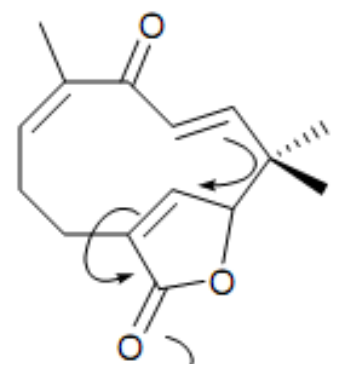

Figure 2 : Structure d'asteriscunolides 3c (Z,E)

\section{Activité antifongique}

Neuf concentrations de deux sesquiterpènes naupliolide et asteriscunolide $3 c$ ont été utilisées pour tester la réponse des trois champignons étudiés (Botrytis cinerea, Penicillium digitatum et Penicillium expansum). Une augmentation des doses des deux produits sesquiterpèniques a été accompagnée par une augmentation de pourcentage d'inhibition (Tableau 3).

Tableau 3 : Pourcentages d'inhibition de la croissance mycélienne de Penicillium digitatum

(PD), Penicillium expansum (PE) et Botrytis cinerea (BC) en utilisant les différentes concentrations de deux sesquiterpènes d'Asteriscus graveolens subsp. odorus.

\begin{tabular}{|c|c|c|c|c|c|c|c|c|c|c|}
\hline & $\begin{array}{l}\text { PI } \\
\text { en } \\
\%\end{array}$ & $\begin{array}{c}\text { 50pp } \\
\text { m }\end{array}$ & $\begin{array}{c}100 p \\
p m\end{array}$ & $\begin{array}{c}125 p \\
\text { pm }\end{array}$ & $\begin{array}{c}150 \mathrm{p} \\
\mathrm{pm}\end{array}$ & $\begin{array}{c}200 p \\
\text { pm }\end{array}$ & $\begin{array}{c}250 p \\
\text { pm }\end{array}$ & $\begin{array}{c}500 \mathrm{p} \\
\mathrm{pm}\end{array}$ & $\begin{array}{c}1000 p p \\
\text { m }\end{array}$ & $\begin{array}{c}\text { 2000pp } \\
\text { m }\end{array}$ \\
\hline \multirow{3}{*}{$\begin{array}{l}\text { Naupli } \\
\text { olide }\end{array}$} & BC & 50,00 & 60,34 & 63,79 & 71,98 & 81,03 & 85,34 & 92,67 & 95,26 & 100,00 \\
\hline & PD & 25,46 & 36,60 & 52,25 & 68,97 & 76,13 & 80,64 & 89,39 & 92,84 & 100,00 \\
\hline & $\mathrm{PE}$ & 29,32 & 39,36 & 53,01 & 65,46 & 72,69 & 85,54 & 93,17 & 98,39 & 100,00 \\
\hline \multirow{3}{*}{$\begin{array}{l}\text { Asteris } \\
\text { cunol- } \\
\text { ide 3c }\end{array}$} & $\mathrm{BC}$ & 24,14 & 36,21 & 45,69 & 81,03 & 84,48 & 92,24 & 97,41 & 100,00 & 100,00 \\
\hline & PD & 27,06 & 39,26 & 45,09 & 53,58 & 70,03 & 87,00 & 89,92 & 92,57 & 93,10 \\
\hline & $\mathrm{PE}$ & 13,65 & 31,73 & 37,75 & 43,78 & 53,82 & 64,26 & 76,71 & 88,76 & 94,78 \\
\hline
\end{tabular}


Test in vitro des sesquiterpènes d'Asteriscus graveolens subsp. odorus sur la croissance mycélienne du Botrytis cinerea

Les deux produits testés naupliolide et asteriscunolide 3c ont affiché des comportements différents en fonction des concentrations utilisées. En effet, si le naupliolide présente de meilleurs résultats en termes d’inhibition de la croissance du Botrytis cinerea à des doses de 50, 100 et 125 ppm estimées par 50, 60,34 et 63,79\%, l'asteriscunolide 3c, quant à lui, se présente plus efficace à des doses de 150 à 1000 ppm estimées de 81,3 à 100\%. Toutefois, à 2000 ppm une inhibition complète similaire des deux produits a été notée (Figure 3 et Tableau 3).

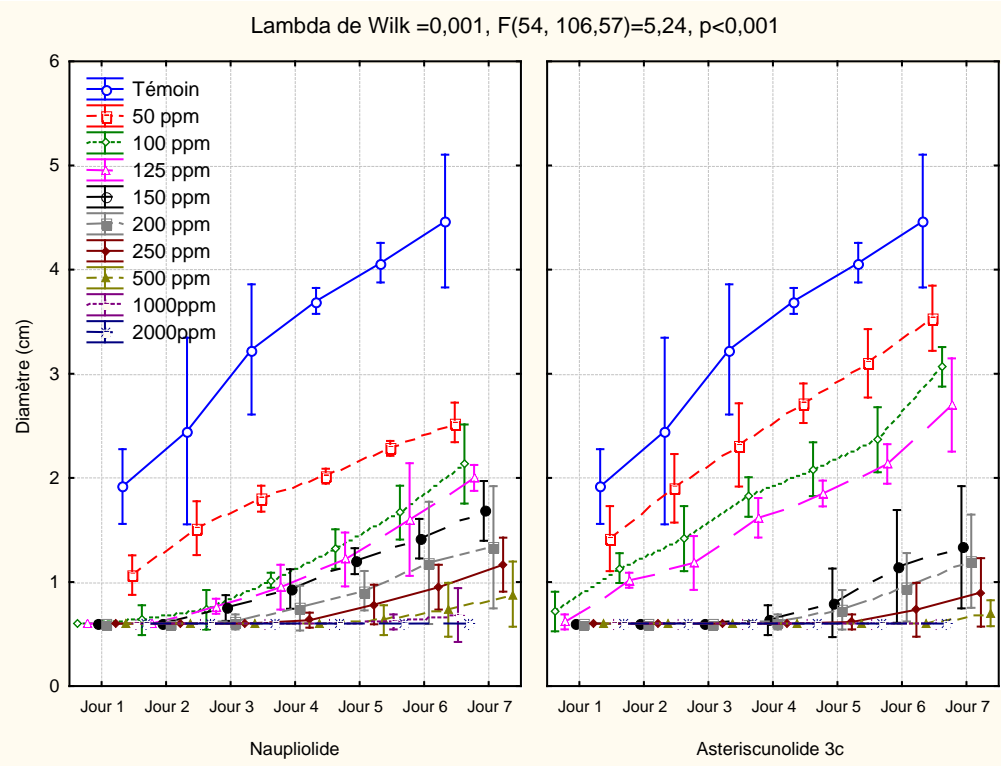

Figure 3 : Évolution de la croissance mycélienne de Botrytis cinerea en fonction de différentes concentrations de deux sesquiterpènes d'Asteriscus graveolens subsp. odorus durant les 7 jours d'incubation.

Test in vitro des sesquiterpènes d'Asteriscus graveolens subsp. odorus sur la croissance mycélienne du Penicillium digitatum

Penicillium digitatum semble répondre sensiblement aux deux produits testés (Figure 4). Toutefois, cette sensibilité est significativement plus accentuée vis-à-vis du naupliolide que de l'asteriscunolide 3c. L'effet amplifié en fonction de la concentration utilisée est toujours remarquable (Tableau 1). 
Lambda de Wilk $=0,0009, F(60,104,602)=4,83, p<0,001$
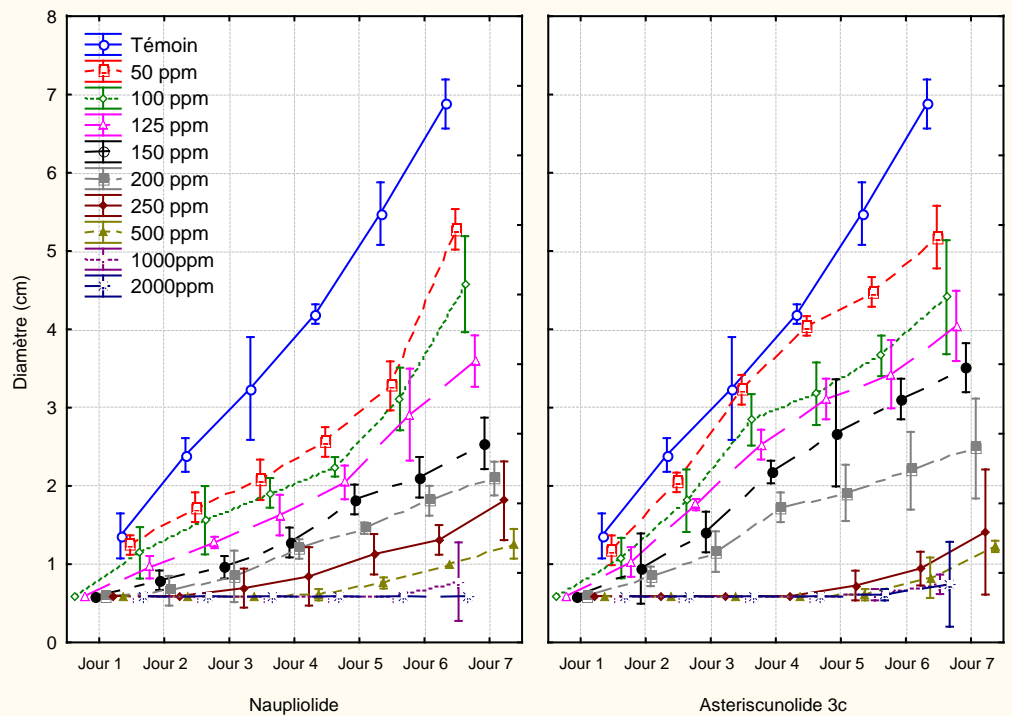

Figure 4 : Évolution de la croissance mycélienne de Penicillium digitatum en fonction de différentes concentrations de deux sesquiterpènes d'Asteriscus graveolens subsp. odorus durant les 7 jours d'incubation.

Test in vitro des sesquiterpènes d'Asteriscus graveolens subsp. odorus sur la croissance mycélienne du Penicillium expansum.

A l'instar du $P$. digitatum, le $P$. expansum répond sensiblement aux deux produits testés, avec une inhibition plus accentuée sous l'effet du naupliolide que de l'asteriscunolide 3c (Figure 5 et Tableau 3).

Lambda de Wilk $=0,0013, F(60,104,602)=4,46, p<0,001$
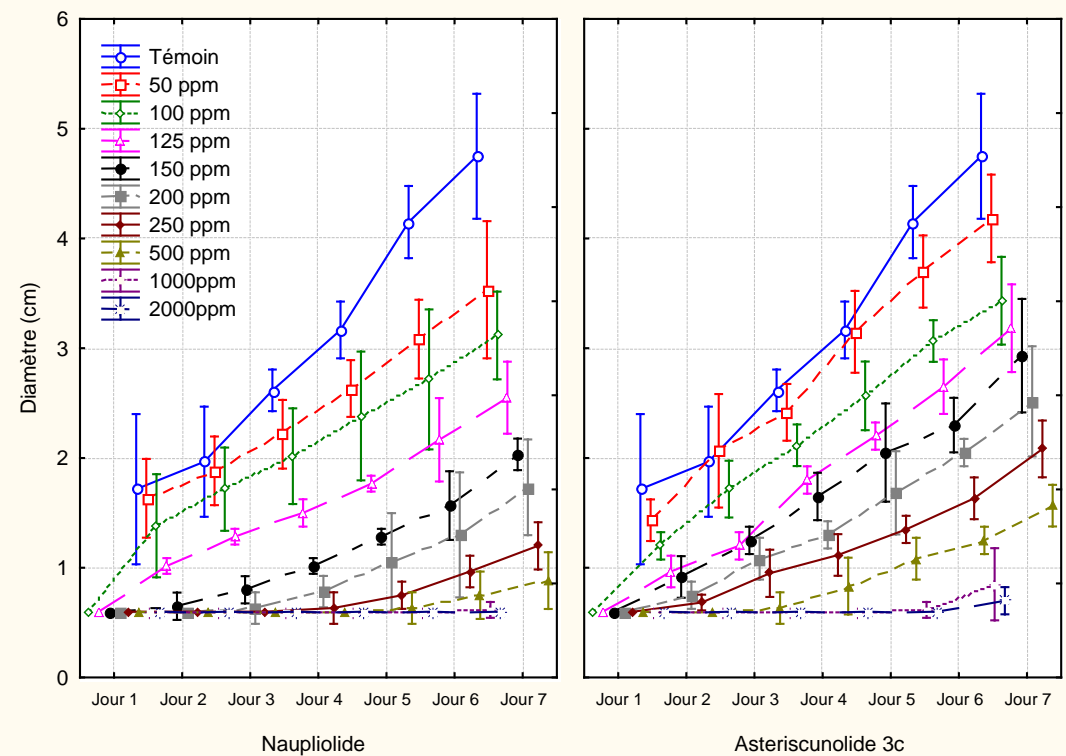

Figure 5 : Évolution de la croissance mycélienne de Penicillium expansum en fonction de différentes concentrations de deux sesquiterpènes d'Asteriscus graveolens subsp. odorus durant les 7 jours d'incubation. 
L'étude de l'activité antifongique des deux sesquiterpènes d'Asteriscus graveolens subsp. odorus a montré une meilleure efficacité sur les trois champignons testés. En effet, le naupliolide, la nouvelle molécule identifiée par Akssira et al. (2006), a marqué une forte inhibition contre Botrytis cinerea à des doses de 50, 100 et 125 ppm et une inhibition complète à la concentration 2000 ppm. Cette même molécule a montré une meilleure efficacité sur Penicillium digitatum et Penicillium expansum. Quant à la molécule asteriscunolide 3c, elle a marqué un effet remarquable sur Botrytis cinerea à des doses de 150 à 1000 ppm et une inhibition complète à la dose 2000 ppm. Toutefois, cette molécule parait moyennement efficace sur Penicillium digitatum et Penicillium expansum.

La recherche de l'activité biologique des lactones sesquiterpèniques a toujours été d'actualité. Ainsi, les propriétés phytotoxiques et cytotoxiques (due à l'existence du groupement $\alpha$-méthylène- $\gamma$-lactone) des asteriscunolides ont fait l'objet d'une étude publiée par Rauter et al. (2001). Cette dernière a montré que, l'asterisunolide 3c présente une activité phytotoxique supérieure à $90 \%$ contre Lemna paucicostata.

Bruneton (1993) a montré que certaines lactones sesquiterpèniques ont une activité antibactérienne contre des germes Gram positifs. C'est le cas de l'hénélanine de l'aunée (Inula helenium) et de la cnicine du chardon béni (Cnicus beniductus).

De même, il a été prouvé que certaines lactones sesquiterpèniques sont antifongiques (Mitscer \& Hasennhuettl, 1975). L'artémisine est un anti malarique qui a donné d'excellents résultats sur l'Homme, tandis que l'ambrosine, un pseudoguaianolide d'Ambrosia maritima, est anthelminthique et molluscide (Bruneton, 1993).

\section{Conclusion}

À l’issue de cette étude nous avons extrait, séparé et identifié deux sesquiterpènes majoritaires à partir d'Asteriscus graveolens subsp. odorus : naupliolide et asteriscunolide 3c. Les structures de ces composés ont été établies sur la base de méthodes spectroscopiques en interprétant les spectres RMN $\left({ }^{1} \mathrm{H},{ }^{13} \mathrm{C}\right.$, COSY, HMQC, HMBC et NOESY) et les spectres IR.

L'étude effectuée pour l'évaluation de l'activité antifongique des deux sesquiterpènes d'Asteriscus graveolens subsp. odorus a montré une différence d'efficacité aussi bien pour les molécules utilisées et les champignons testés que les différentes concentrations étudiées. En effet, cette étude a révélé une forte inhibition du naupliolide contre Botrytis cinerea, Penicillium digitatum et Penicillium expansum. Quant à la molécule asteriscunolide 3c, elle a marqué un effet remarquable sur Botrytis cinerea. Toutefois, cette dernière molécule semble être moyennement efficace sur Penicillium digitatum et Penicillium expansum. 
L’efficacité des sesquiterpènes majoritaires de la plante Asteriscus graveolens subsp. odorus pourrait être le sujet d'une investigation et une exploitation dans la lutte intégrée contre les trois champignons testés qui causent beaucoup de dégâts sur les fruits et légumes en post-récolte : Botrytis cinerea, Penicillium digitatum et Penicillium expansum.

\section{References:}

1. Adams RP., 2001. Identification of essential oil components by Gas Chromatography. Quadrupole Mass Spectroscopy. Allured Publishing Corp. Carol Stream (III, USA).

2. Ahmed AA., 1992. Farnesol and thymol derivatives from Asteriscus pygmaeus. J. of Nat. Prod. 55, 824-827.

3. Alilou H., Talbi J. \& Idrissi Hassani LM., 2013. Activité antifongique des extraits de quatre plantes du sud du Maroc : Asteriscus imbricatus (Cav.) DC., Asteriscus graveolens subsp. odorus (Schousb.) Greuter, Cladanthus arabicus (L.) Cass. et Kleinia anteuphorbium (L.) Haw. Science Lib. 5, 1-9.

4. Alilou H., Rouhi R., Idrissi Hassani LM \& Akssira M., 2007. Activité antifongique de Bubonium odorum (Astéracées) sur des champignons pathogènes d'agrumes. Reviews in Biology and Biotechnology. 6 (1), 20-24.

5. Akssira M., Mellouki F., Salhi A., Alilou H., Saouf A., El Hanbali F., Jesus, FA. \& Barrero AF., 2006. Naupliolide, a sesquiterpene lactone with a novel tetracyclic skeleton from Nauplius graveolens sub sp. odorus. Tetrahedron Letters. 47, 6719-6721.

6. Bohlmann F. \& Pauls A., 1992. Humulane derivatives from Nauplius intermedius Phytochemistry. 31, 1663-1665.

7. Bruneton J., 1993. Phytochimie et pharmacognosie des plantes médicinales, éditions techniques et documentations Lavoisier. 915 p.

8. El-Dahmy S., Jakupovic J., Bohlmann F. \& Sarg TM., 1985. New humulene derivatives from Asteriscus graveolens. Tetrahedron Letters. 41 (2), 309-316.

9. Jakupovic J., Lehmann L., Bohlmann F. \& Hogdson AA., 1987. Nerolidol derivatives from Asteriscus sericeus. Phytochemistry. 26 (10), 2854-2855.

10. Mitscer LA. \& Hasennhuettl G., 1975. Phytochemistry. 9, 243-283.

11. Rauter AP., Branco I., Bermejo J., Gonzalez AG., Garcia-Gravalos MD. \& San Feliciano A., 2001. Bioactive humulene derivatives from Asteriscus vogelii. Phytochemistry. 56, 167-171.

12. San Feliciano A., Barrero AF., Medarde M., Del Corral JM., Ledesma E. \& Sanchez-Ferrando F., 1982. Humulanolide from Asteriscus aquaticus. Tetrahedron Letters. 23, 3097-3100. 
13. San Feliciano A., Barrero AF., Medarde M., Del Corral JM., Aizpiri AA. \& Sanchez-Ferrando F., 1984. Astericunolides A, B, C and D, the first humulanolides; two pairs of conformationally stable stereoisomers. Tetrahedron Letters. 40, 873-878.

14. San Feliciano A., Barrero AF., Medarde M., Del Corral JM., Aramburu A. \& Perales A., 1985a. The stereochemistry of asteriscunolides. An X-Ray based correction. Tetrahedron Letters. 41, 5711-5717.

15. San Feliciano A., Barrero AF., Medarde M., Del Corral JM., Aramburu A., Perales A. \& Fayos J., 1985b. Asteriscunolide A : sesquiterpene lactone with a new natural skeleton. Tetrahedron Letters. 26, 2369-2382.

16. San Feliciano A., Medarde M., del Corral JM., Aramburu A., Gordaliza M. \& Barrero AF., 1989. Aquatolide A new type of humulane-related sesquiterpene lactone. Tetrahedron Letters. 30, 2851-2854. 